\title{
Sicherung des Eigenkapitals bei der gesetzestypischen Kommanditgesellschaft
}

\author{
INGO KOLLER
}

\section{Problem}

\section{Vorbemerkung}

In den siebziger und achtziger Jahren ist die Sicherung des Eigenkapitals von Gesellschaften in den Vordergrund der Diskussion getreten. Man braucht nur an die Stichworte eigenkapitalersetzende Darlehen, Haftung bei Unterkapitalisierung, objektive Bestimmung des Eigenkapitalcharakters von Gesellschaftermitteln ${ }^{1}$ und mit Einschränkungen an das Stichwort der Konzernhaftung zu erinnern. Die Diskussion erstreckte sich zunächst schwerpunktmäßig auf die $\mathrm{GmbH}$ und die $\mathrm{GmbH} \&$ Co KG; sie hat inzwischen aber auch die gesetzestypische KG erreicht.

\section{Vorschläge zur Verbesserung der Eigenkapitalbildung und-sicherung}

a) Extensive Auslegung des Begriffs der Einlagenrückgewähr

Bekanntlich verwendet der Gesetzgeber den Begriff der Einlage in den \S $161 \mathrm{ff}$ HGB mehrdeutig ${ }^{2}$. Der Begriff kann auf die im Handelsregister eingetragene Einlage, die Haftsumme, bezogen sein. Unter Einlage kann man aber auch den im Gesellschaftsvertrag unter den Gesellschaftern versprochenen Beitrag, die sog. Pflichteinlage, verstehen. Weil die für das Innenverhältnis unter den Gesellschaftern bedeutsame Pflichteinlage und die im Außenverhältnis zu den Gesellschaftsgläubigern unmittelbar maßgebliche Haftsumme auseinanderzuhalten sind, können Pflichtein-

' Vgl. BGH, NJW 1980, 1522, 1523; 1981, 2251, 2252; 1982, 2253, 2254; GmbHRdSch. 1984, 37; dazu Joost, ZGR 1987, 370, 396 ff; H.P. Westermann, FS Fleck, ZGR-Sonderheft Nr.7, S. 423, 425; Schön, ZGR 1990, 228 ff; K. Schmidt, Gesellschaftsrecht (1986), $\$ 18$ III 3 .

2 Dies war dem HGB-Gesetzgeber bekannt (Denkschrift zum Entwurf eines HGB; Hahn/Mugdan, Die gesammten Materialien zu den Reichsjustizgesetzen, Bd. 6 [Neudruck 1983], S. 285). 
lage und Haftsumme in unterschiedlicher Höhe festgesetzt werden ${ }^{3}$. Vor allem bei Publikumskommanditgesellschaften wurde häufig eine die Haftsumme übersteigende Pflichteinlage vereinbart.

Je nachdem, ob man den Begriff der Einlage in $\$ 172$ IV 1 HGB im Sinn der Pflichteinlage oder im Sinn der Hafteinlage versteht, kommt man in Fällen der Einlagenrückgewähr zu ganz unterschiedlichen Ergebnissen, wenn der Betrag der Pflichteinlage die Haftsumme übersteigt.

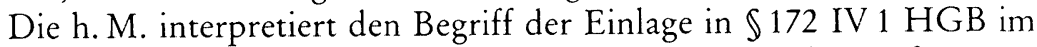
Sinne der Haftsumme. Rückzahlungen, die nur die die Haftsumme überschießende Pflichteinlage schmälern, führen deshalb nach h.M. nicht zum Wiederaufleben der Haftung. ${ }^{4}$. Die die Haftsumme übersteigende Pflichteinlage bildet also, soweit sie nicht bereits durch Verluste vebraucht worden ist, eine Art Polster, das vor dem Wiederaufleben der unmittelbaren Kommanditistenhaftung gegenüber den Gesellschaftsgläubigern schützt.

Karsten Schmidt ${ }^{5}$ hält dieser Interpretation entgegen, daß sie die Kapitalgarantien schwäche, die die Gläubiger schützen. Bei der KG gebe es anders als bei der $\mathrm{GmbH}$ kein summenmäßig gesichertes Garantiekapital. Deshalb müßten Gläubiger schon ab der Rückzahlung der ersten Mark Pflichteinlage ohne Rücksicht auf die Höhe der Haftsumme gemäß $\int \$ 172$ IV 1, 171 I HGB haften. Der Kapitalerhaltungsgrundsatz bei der KG gewährleiste nämlich den Bestand derjenigen Haftungsmasse, die sich aufgrund der Gesellschaftereinlagen tatsächlich gebildet habe.

\section{b) Wiederaufleben der Haftung über den Betrag der Haftsumme hinaus}

Immerhin ist auch Karsten Schmidt der Ansicht, daß die Haftung gemäß den $\$ \$ 172$ IV 1, 171 I HGB nur bis zum Betrag der Haftsumme wieder auflebt, selbst wenn eine die Haftsumme übersteigende Pflichteinlage oder sogar ein diese Pflichteinlage übersteigender Vermögenswert zurückgewährt worden ist ${ }^{6}$. Dies entspricht der ganz herrschenden Meinung? ${ }^{7}$ Oberste Grenze der erneuten Haftung ist die Haftsumme, selbst wenn ein Mehrfaches der Haftsumme zurückgeflossen ist. Der

3 Dies hielt schon der historische Gesetzgeber für zulässig. Vgl. Denkschrift (Fn. 2), S. 285 .

+ U. Huber, ZGR 1988, 1, 13;RGRK z. HGB-Weipert (1950), \$172 Anm. 30; Schlegelberger/Geßler, HGB (4.Aufl.), \$172 Anm. 16; Staub/Schilling, Großkommentar HGB (4. Aufl.), $\$ 172$ Rdn. 10; Heymann/Horn, HGB (1989), \172 Rdn. 15.

5 K. Schmidt, Einlage und Haftung des Kommanditisten (1977), S. $79 \mathrm{ff}$; Schlegelberger/K.Schmidt, HGB (5. Aufl.), $\$ \$ 171,172$ Rdn. 64.

${ }^{6}$ K. Schmidt, Einlage (Fn. 5), S. 80.

BGHZ 60, 324, 332; U.Huber, ZGR 1988, 1, 14; Rümker, ZGR 1988, 494, 509; Lutter/Hommelhoff, ZGR 1979, 31, 45; RGRK z. HGB-Weipert (2. Aufl. 1950), $\$ 172$ 
$\mathrm{BGH}^{8}$ begründet die Sachgerechtigkeit dieser Obergrenze damit, daß das Gläubigergefährdungspotential bei der gesetzestypischen Kommanditgesellschaft im Vergleich zu Gesellschaften mit umfassend beschränkter Haftung bedeutend geringer sei. Die volle Haftung des Komplementärs wirke nämlich als Bremse, weil der Komplementär im eigenen Interesse den Kapitalwünschen des Kommanditisten entgegentreten werde, um sein Haftungsrisiko nicht übermäßig ansteigen zu lassen. Außerdem werde der Komplementär wegen seiner eigenen unbeschränkten Haftung die Geschäfte der Gesellschaft in erhöhtem Maße vorsichtig füh-

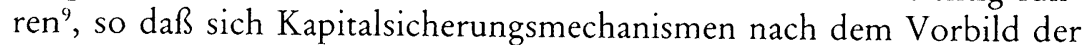
$\mathrm{GmbH}$ erübrigten.

Von diesem Standpunkt aus ist denn auch mit der neueren Kommentarliteratur anzunehmen, daß der Kommanditist, der seine Einlage in Höhe der Haftsumme noch nicht geleistet hat, selbst dann nur bis zum Betrag der Haftsumme haftet, wenn er sich sämtliche Gewinne der KG hat auszahlen lassen, Verluste aber nicht ausgeglichen hat, so daß sein Kapitalkonto bei der Ausschüttung der Gewinne negativ war ${ }^{10}$.

Gegen diese Interpretation des $\$ 172$ IV 1 HGB hat sich Joost ${ }^{11}$ gewandt. Er vertritt die Meinung, daß der Gläubigerschutz bei der KG dem der $\mathrm{GmbH}$ anzugleichen sei. Bei der $\mathrm{GmbH}$ würden etwaige Rückzahlungsansprüche der $\mathrm{GmbH}$ gemäß $\$ \$ 30,31 \mathrm{GmbHG}$ weder durch den Betrag der Stammeinlage noch durch den Betrag des Stammkapitals beschränkt, wenn an Gesellschafter aus dem GmbH-Vermögen Leistungen geflossen seien, die zu einer Unterbilanz der GmbH geführt bzw. sie vertieft haben. In ähnlicher Weise soll nach der Ansicht Joosts auch der Kommanditist haften, der von der KG Zahlungen erhalten hat, durch die sein Kapitalkonto - weiter - unter den Betrag der Haftsumme gemindert worden ist. Joost begründet seine These im wesentlichen mit zwei Argumenten: Unter Einlage im Sinn des $\$ 172$ IV 1 HGB sei in Parallele zum Begriff des Stammkapitals die bilanzielle Kapitalziffer zu verstehen, die angebe, welcher Teil des Vermögens zugunsten der Gläubiger gebunden sei. Die Funktionen von Stammkapital bei der GmbH und von Einlage bei der KG seien mithin identisch. Zum anderen sei die Verweisung des $\$ 172$ IV 1 HGB auf $\$ 171$ I HGB mit der Folge des Wiederauflebens der unmittelbaren Haftung des Kommanditisten nicht

Anm. 33; Schlegelberger/Geßler (Fn. 4) \$172 Anm. 13; Schlegelberger/K. Schmidt (Fn. 5), $\int \$ 171,172$ Rdn. 65; Staub/Schilling, HGB (Fn. 4) \$172 Rdn. 10; Heymann/Horn, HGB (Fn. 4), \172 Rdn. 15; Westermann u.a., Handbuch der Personengesellschaften I 927.

8 BGHZ 60, 324, 332; BGH, ZIP 1990, 578, 583.

? BGH, ZIP 1990, 578, 583.

10 Schlegelberger/K. Schmidt (Fn. 5), \$\$171, $172 \mathrm{Rdn} .79$.

"Joost, ZGR 1987, 370, $382 \mathrm{ff}$. 
Ausdruck einer personengesellschaftsrechtlichen Besonderheit, sondern nur Ausdruck eines technischen Kunstgriffs. Aus der Sicht der Gläubiger sei es nämlich nicht so wichtig, ob sie dadurch geschützt würden, daß der Gesellschafter wie bei der $\mathrm{GmbH}$ gläubigergefährdende Auszahlungen an die Gesellschaft zurückzugewähren habe oder dadurch, daß sie, die Gläubiger, unmittelbar auf das Privatvermögen der Gesellschafter, der Kommanditisten, zugreifen könnten. Entscheidend sei allein der Umfang des Zugriffs. Habe ein Kommanditist das Vermögen der KG über den Betrag der Haftsumme hinaus geplündert, so sei jedenfalls die Begrenzung der Haftung auf die Haftsumme nicht mehr angemessen. Joost ${ }^{12}$ schlägt deshalb vor, daß der Kommanditist analog den $\$ \$ 172$ IV 1, 171 I HGB auf den vollen Wert der empfangenen Leistung ohne Rücksicht auf die Höhe der Haftsumme zu haften habe. Eine Ausnahme solle nur in den Fällen gelten, in denen trotz der Leistung genügend Aktivvermögen in der KG verblieben sei, um damit die echten Passiva der KG zuzüglich der Haftsummen der Kommanditisten abzudecken.

\section{c) Eigenkapitalersetzende Darlehen}

aa) Karsten Schmidt hat in mehreren Stellungnahmen die Ansicht vertreten, daß die für die $\mathrm{GmbH}$ und die $\mathrm{GmbH} \& \mathrm{CoKG}$ entwickelten Regeln über die Behandlung eigenkapitalersetzender Darlehen im wesentlichen auch auf die KG mit einer natürlichen Person als Komplementär anzuwenden seien; denn bei den Gesellschafterdarlehen, die einer Gesellschaft statt des notwendigen Eigenkapitals gewährt werden, handle es sich um ein rechtsformübergreifendes Haftungs- und Finanzierungsproblem, das nicht notwendig mit einer umfassend beschränkten Haftung zusammenhänge ${ }^{13}$. Entscheidend sei vielmehr, daß eine Gesellschaft, die auf dem freien Kreditmarkt bei Offenlegung aller Umstände kein Fremdkapital erhalten würde, objektiv Eigenkapital benötige, und daß die Gesellschafter, die ihr in dieser Situation Kapital zuführten, dieses Kapital auch im Konkurs als Eigenkapital und nicht als Fremdkapital behandeln lassen müßten. $₫ 32$ a GmbHG und $\$ 172$ a HGB seien deshalb als Ausprägung eines allgemeinen Finanzierungsgrundsatzes anzusehen.

Habe sich aber ein Kommanditist schon vor dem Konkurs der KG das Darlehen zurückzahlen lassen, so könne man nicht einfach eine Analogie zu den $\$ \$ 30 \mathrm{ff}$ GmbHG, 172 a HGB ziehen, da es im Recht der gesetzestypischen KG anders als im Recht der GmbH und GmbH \& Co

12 Joost, ZGR 1987, 370, 391.

13 K. Schmidt, AG 1984, 12, 14; GmbHRdSch. 1986, 337, 340; ZGR 1986, 152, $159 \mathrm{ff}$; JZ 1985, 302, 304; vgl. auch Kollhosser, WM 1985, 932. 
KG nicht einmal in Hinblick auf echte Einlagen ein Auszahlungsverbot $^{14}$ gebe. Deshalb lasse sich auch in Hinblick auf eigenkapitalersetzende Darlehen kein Auszahlungsverbot begründen ${ }^{15}$. Eine Analogie zu \172 IV HGB scheide aus, weil die Haftung nur bis zur Haftsumme wieder aufleben könne, während die eigenkapitalersetzenden Darlehen häufig weit über dem Betrag der Haftsumme lägen. Außerdem sei eine Haftung analog $\$ 172$ IV $H G B$ zu streng ${ }^{16}$. Sie führe nämlich zu einer erneuten Haftung ohne zeitliche Beschränkung. Karsten Schmidt plädiert daher in Hinblick auf zurückgezahlte eigenkapitalersetzende Gesellschafterdarlehen dafür, eine Analogie zu $\$ 237$ HGB zu ziehen ${ }^{17}$.

bb) Joost ${ }^{18}$ geht dagegen, gestützt auf seine These, daß das KG-Vermögen gegen Vermögensabflüsse in Richtung auf die Kommanditisten über die Haftsummen hinaus analog $\$ 172$ IV HGB geschützt sei'19, einen Schritt weiter. Auch er betont, daß die Gründe, die für eine Gleichstellung von eigenkapitalersetzenden Darlehen und echtem Eigenkapital sprächen, nicht spezifisch GmbH-rechtlicher Natur seien. Das gelte sowohl für den topos des widersprüchlichen Verhaltens ${ }^{20}$ als auch für das Kriterium der mangelnden Kreditwürdigkeit der Gesellschaft in den Augen Dritter ${ }^{21}$. Es sei deshalb geboten, die sich bei der $\mathrm{GmbH}$, der $\mathrm{GmbH} \& \mathrm{Co} \mathrm{KG}$ und der gesetzestypischen KG gleichartig stellenden Probleme gleich zu behandeln. Der Umstand, daß der Kommanditist keine Mindesteinlage zu erbringen habe, stehe ebensowenig einer Übernahme der $\mathrm{GmbH}$-Regeln des eigenkapitalersetzenden Darlehens entgegen wie die Tatsache, daß ein Komplementär persönlich voll hafte; denn der Komplementär könne vermögenslos sein, und jeder Kommanditist habe immerhin mit einem gewissen Betrag zu haften ( $\$ 171 \mathrm{f} \mathrm{HGB}$ ). Analog diesen Haftungsregeln müsse der Kommanditist sein Privatvermögen dem Zugriff der Gesellschaftsgläubiger öffnen, wenn er sich eigenkapitalersetzende Gesellschafterdarlehen habe zurückzahlen lassen, obwohl dadurch eine Unterbilanz herbeigeführt oder verstärkt worden sei. Gegen den Vorwurf, eine analoge Anwendung des $\$ 172$ IV HGB auf die Rückzahlung eigenkapitalersetzender Darlehen setze sich in Wider-

14 Schlegelberger/K.Schmidt, HGB (Fn.5), $\$ \mathbb{S} 171,172 \mathrm{Rdn} .62$; Staub/Schilling, HGB (Fn. 4), $\$ 172$ Rdn. 9.

15 K. Schmidt, GmbHRdSch. 1986, 337, 340.

${ }_{16}$ K.Schmidt, GmbHRdSch. 1986, 337, 342; ebenso Rümker, ZGR 1988, 494, 511.

17 K. Schmidt, GmbHRdSch. 1986, 337, 342.

is Joost, ZGR 1987, 370, $393 \mathrm{ff}$.

19 Siehe oben bei Fn. 11.

$20 \mathrm{Vgl}$. BGHZ 31, 258, 272 f; 75, 334, $336 \mathrm{f}$.

21 Vgl. BGHZ 76, 326, 330; K. Schmidt, Gesellschaftsrecht (1986), S. 395. 
spruch zu $\$ 172$ a HGB, der die $\$ \$ 32$ a, 33 b GmbHG nur auf KGs ohne eine natürliche Person als Komplementär für anwendbar erklärt habe, führt Joost das Argument ins Feld, daß die Systemeinheit nicht wichtiger sein dürfe als das Ziel des Gläubigerschutzes und das Ziel einer allgemein gerechten Lösung des Problems der eigenkapitalersetzenden Darlehen ${ }^{22}$. Allerdings überträgt Joost die GmbH-rechtlichen Regeln der eigenkapitalersetzenden Darlehen nicht voll auf die gesetzestypische KG. Er begründet dies damit, daß bei der $\mathrm{GmbH}$ ein Auszahlungsverbot bestehe, während es bei der gesetzestypischen Kommanditgesellschaft den Gesellschaftern offenstehe, im Innenverhältnis die Einlage und damit erst recht eigenkapitalersetzende Darlehen an den Kommanditisten zurückzuzahlen bzw. rückzahlbar zu stellen ${ }^{23}$. Beim Darlehen könne daher ein Rückzahlungsverbot nur auf das Treuegebot gestützt werden ${ }^{2+}$.

Diesen Vorschlägen, die Kapitalsicherung bei der KG zu verbessern, hat U.Huber entgegengehalten, daß Darlehen nur zu Lasten solcher Gesellschafter dem Eigenkapital gleichgestellt werden dürften, die Einfluß auf die Unternehmensleitung besäßen. Dies sei bei dem typischen Kommanditisten nicht der Fall.

\section{d) Stille Beteiligung}

Schön ${ }^{26}$ kritisiert die Rechtsprechung des BGH, derzufolge die Einlagen stiller Gesellschafter als Eigenkapital zu qualifizieren sind, falls die stillen Gesellschafter in der KG kommanditistenähnliche Positionen einnehmen würden, als „richterlichen Oktroi“, weil diese Qualifikation dem klaren rechtsgeschäftlichen Willen der Gesellschafter zuwiderlaufe.

Schön $n^{27}$ stimmt aber dem BGH weitgehend im Ergebnis zu. Er schlägt vor, sich zu einer besseren Begründung auf die Wurzeln der stillen Gesellschaft zu besinnen. Aus historischer Sicht liege der Unterschied zwischen der KG und der stillen Gesellschaft darin, daß der Kommanditist anders als der stille Gesellschafter die öffentliche Zusage gemacht habe, mit seiner Einlage für das Unternehmen einstehen zu wollen. Der Grundsatz, daß die Offenlegung der Einlage zur Haftung mit der Einlage als Eigenkapital führe, sei durch die Ausdifferenzierung von KG und stiller Gesellschaft im HGB nicht obsolet geworden. Lege ein stiller Gesellschafter eines Einzelkaufmanns seine Beteiligung offen, so dürfe

22 Joost, ZGR 1987, 370, 401; ebenso i. E. K. Schmidt, AG 1984, 12, 14; a. A. OL.G Düsseldorf, WM 1983, 874; Rümker, ZGR 1988, 494, 512.

23 Rümker, ZGR 1988, 494, 509.

24 Joost, ZGR 1987, 370, 396.

25 U. Huber, ZGR 1988, 1, 40.

2t Schön, ZGR 1990, 220, 241.

27 Schön, ZGR 1990, 220, $234 \mathrm{ff}$. 
zwar heute darin keine Haftungszusage gesehen werden, weil das Vermögen des Einzelkaufmanns keinem Kapitalaufbringungs- und -erhaltungsmechanismus unterworfen sei. Die Gläubiger könnten daher die Offenlegung der Beteiligung nicht als rechtlich bindende Stärkung des Betriebskapitals auffassen. Anders sei jedoch die Situation bei KGs, deren Finanzierung im wesentlichen über Kapitaleinlagen von Anlegern erfolge. In diesen Fällen könne die Offenlegung der Einlage, z. B. in Anlageprospekten, als Haftungszusage verstanden werden; denn in diesen Fällen sei die Finanzierung von wirtschaftlich zentraler Bedeutung. Maßgeblich sei der Empfängerhorizont des Rechtsverkehrs. Wenn in einem Zeitpunkt, in dem die KG nicht - mehr - kreditwürdig ${ }^{28}$ sei, die Zeichnung einer stillen Einlage offenbart werde, so sehe der Rechtsverkehr darin eine Erweiterung des Kapitalstocks und nicht bloß die Zufuhr von Fremdkapital. Es genüge, daß die stille Beteiligung einer einzigen Person gegenüber aufgedeckt worden sei; denn die Qualifikation als Eigenkapital müsse allen Gläubigern gegenüber einheitlich erfolgen. Unerheblich sei es, ob gleichzeitig der gesellschaftsvertragliche Ausschluß der Verlustbeteiligung offengelegt werde; denn dies sei beim Kommanditisten auch nur im Innenverhältnis von Bedeutung.

Selbst in Fällen, in denen Kommanditisten zusätzlich zu ihrer Kommanditeinlage stille Beteiligungen zeichnen, ohne dies publik zu machen, muß Schön ${ }^{29}$ zufolge in der Vereinbarung einer stillen Einlage die Zusage einer Erweiterung des Eigenkapitaleinsatzes gesehen werden, wenn das Kapitalkonto des Kommanditisten bei Zeichnung der stillen Einlage negativ gewesen sei. Dann habe nämlich die objektive Notwendigkeit einer Kapitalzufuhr bestanden. Die stille Einlage könne daher nur unter der Sanktion des $\$ 172$ IV HGB zurückgewährt werden. Die Situation ähnle hier den Fällen, in denen der Kommanditist, dessen Kapitalkonto negativ geworden ist, eine zusätzliche Pflichteinlage gezahlt habe und sich diese nach der Verrechnung mit den angelaufenen Verlusten zurückgewähren lasse. In diesen Fällen lebe nach allgemeiner Meinung die Haftung gemäß \$172 IV HGB wieder auf. Nicht anders könne die Situation bei der Gewährung einer stillen Einlage statt der Erhöhung der Pflichteinlage sein.

\section{Stellungnahme}

\section{Begriff der Einlage in $\$ 172$ IV HGB}

$\mathrm{Ob}$ der historische Gesetzgeber bei Verwendung des Begriffs der Einlage eher an die Haftsumme oder an die Pflichteinlage gedacht hat,

28 Wiedemann, FS Bärmann (1975), S. 1037, 1038; U.Huber, ZGR 1988, 1, 16; Lutter/Hommelhoff, ZGR 1979, 31, 45.

29 Schön, ZGR 1990, 220, $244 \mathrm{ff}$. 
läßt sich den Materialien zum $\mathrm{HGB}^{30}$ nicht eindeutig entnehmen. Man ist daher auf das Instrument der systematischen und teleologischen Auslegung angewiesen.

Aufschlußreich ist vor allem ein Vergleich der $\$ \$ 172$ III HGB, 174

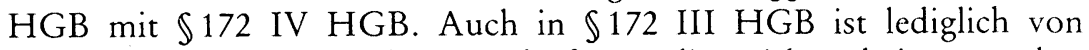
Einlage die Rede. Weil die Vorschrift nur die Wirksamkeit gegenüber den Gläubigern regeln will, wird zu Recht allgemein angenommen, daß unter Einlage die im Handelsregister eingetragene Haftsumme zu verstehen ist ${ }^{31}$. Gleiches gilt für $\$ 174 \mathrm{HGB}$, der mit dem $\$ 172$ III HGB nahe verwandt ist. Auch in $\$ 174 \mathrm{HGB}$ spricht der Gesetzgeber nur schlechthin von Einlage. Der Umstand, daß gemäß $\$ 174$ HGB die Herabsetzung der Einlage von der Eintragung im Handelsregister abhängig sein soll, im Handelsregister aber nur die Haftsumme, nicht aber die Pflichteinlage einzutragen ist, zeigt, daß das HGB in $\$ 174$ HGB mit Einlage ebenfalls ausschließlich die Haftsumme meint ${ }^{32}$. Aus $\$ 174$ HGB ergibt sich mithin, daß die Haftsumme mit Wirkung gegenüber den Gläubigern zwar nicht erlassen, aber unter bestimmten Voraussetzungen herabgesetzt werden darf. Im Umkehrschluß bedeutet dies, daß eine die Haftsumme übersteigende Pflichteinlage beliebig ermäßigt ${ }^{33}$ und im Verhältnis unter den Gesellschaftern sogar in vollem Umfang erlassen werden darf ${ }^{34}$; denn die $\mathbb{S} 172$ III, 174 HGB schützen die Gläubiger nur vor dem gänzlichen Erlaß bzw. eingeschränkt vor der Herabsetzung der Haftsumme. Falls nun aber die Pflichteinlage sogar ganz erlassen werden darf, ohne daß die Gläubiger hieraus Rechte ableiten können, so ist es nur konsequent, daß die Gläubiger auch dann keine Rechte gegenüber den Kommanditisten geltend machen dürfen, wenn die Gesellschafter beschließen, die Pflichteinlage bis zur Höhe der Haftsumme herabzusetzen. Erlaubt man die haftungsunschädliche Herabsetzung der Pflichteinlage, so muß es auch gestattet sein, die Beträge, die der Kommanditist der KG nicht mehr als Pflichteinlage zur Verfügung stellen muß, haftungsunschädlich zurückzuzahlen ${ }^{35}$.

30 In der Denkschrift zum Entwurf eines HGB (Hahn/Mugdan, Die gesammten Materialien zu den Reichsjustizgesetzen, Bd. 6 [Neudruck 1983], S. 285) hebt der Geset\%geber nur hervor, daß es besonderer Vorschriften über die Höhe, die Erhöhung und Herabsetzung der Einlage bedürfe, weil die Einlage im Innenverhältnis nicht unbedingt mit dem Betrag übereinstimmen müsse, der für das Verhältnis zu den Gläubigern maßgeblich sei.

31 Denkschrift (Fn. 30), S. 285; Staub/Schilling, HGB (Fn.4), \$172 Rdn. 1.

32 Staub/Schilling, HGB (Fn. 4), $\$ 174$ Rdn. 1.

33 So auch K.Schmidt, ZGR 1986, 152, 161; Staub/Schilling, HGB (Fn. 4), $\$ 174$ Rdn. 1.

34 Staub/Schilling, HGB (Fn. 4), \$172 Rdn. 7.

35 Ähnlich U. Huber, ZGR 1988, 1, 13, Fn. 36. 
Eine Verstärkung des Gläubigerschutzes durch eine extensive Interpretation des Begriffs der Einlage in $\$ 172$ IV HGB ist auch ineffizient, wenn man daran nur die Rechtsfolge knüpft, daß die Rückgewähr zum Wiederaufleben der Haftung bis zur Grenze der Haftsumme führt. Die Gläubiger erhalten nämlich gegenüber Kommanditisten, denen eine die Haftsumme übersteigende Pflichteinlage zurückgezahlt wird, nur so lange erhöhten Schutz, solange die Kommanditisten nicht gelernt haben, sich auf diese Rechtsansicht einzustellen. Haben sie aber begriffen, daß ihre erneute Haftung maximal den Betrag der Haftsumme erreichen wird, so werden sie ihre ganze Einlage abziehen und eine erneute Haftung mit der Haftsumme ${ }^{36}$ in Kauf nehmen. Der die Haftsumme überschießende Betrag der Pflichteinlage ist nämlich an sie haftungsfrei zurückgelangt. Von der Haftung mit der Haftsumme können sie sich durch Zahlung an einen Gesellschaftsgläubiger ${ }^{37}$ oder durch erneute Einzahlung auf die herabgesetzte ${ }^{38}$ Pflichteinlage ${ }^{39}$ befreien. Auch dies zeigt, daß man unter Einlage im Sinn des $\$ 172$ IV 1 HGB nur die Haftsumme verstehen kann. Die die Haftsumme übersteigende Pflichteinlage bildet mithin für haftungsunschädliche Rückzahlungen eine Art Polster, allerdings nur unter der Voraussetzung, daß dieses Polster nicht bereits durch Verluste verbraucht worden ist.

Es kann somit festgehalten werden, daß die Eigenkapitalsituation der KG nicht mit Hilfe einer extensiven Auslegung des Begriffs der Einlage verbessert werden kann ${ }^{40}$.

\section{Haftung über die Grenze der Haftsumme hinaus}

Gemäß dem Wortlaut des $₫ 172$ IV HGB in Verbindung mit $\$ 171 \mathrm{I}$ HGB lebt die Haftung maximal in Höhe der Haftsumme wieder auf. Das gilt auch in Fällen, in denen ein Mehrfaches der Haftsumme zurückgewährt wird und dem Kommanditisten dadurch die Möglichkeit eröffnet wird, seine Haftung wirtschaftlich gesehen trotz Rückzahlung seiner Einlage abzuschütteln. Bekommt er nämlich das Doppelte seiner Haftsumme zurück, so kann er die eine Hälfte des zurückgezahlten Betrages ohne Zugriffsmöglichkeit der Gesellschaftsgläubiger für sich verwerten, während er die andere Hälfte dazu nutzen kann, an Gesell-

${ }^{36}$ Es wird hier mit der h.M. (Fn. 7) davon ausgegangen, daß die Haftung unabhängig von der Höhe der Rückzahlung nur bis zum Betrag der Haftsumme wiederauflebt.

37 BGHZ 51, 393.

${ }^{38}$ In der Rückzahlung liegt in der Regel eine zumindest vorübergehende Herabsetzung der Pflichteinlage.

${ }^{39}$ Es bleibt offen, ob der Einlage- oder der Verrechnungstheorie zu folgen ist (vgl. Schlegelberger/K.Schmidt, HGB [Fn. 5], \$\$ 171, 172 Rdn. 46).

to So i.E. die ganz herrschende Meinung (vgl. Fn. 4). 
schaftsgläubiger haftungsbefreiend zu leisten ${ }^{41}$. Dieses Ergebnis vermag sicher nicht recht einzuleuchten.

Die Beschränkung der wiederauflebenden Haftung auf die Haftsumme steht auch kaum mit der $\$ 172$ IV 2 HGB zugrundeliegenden ratio legis im Einklang. Gemäß $₫ 172$ IV 2 HGB lebt die Haftung des Kommanditisten nämlich wieder auf, soweit ein Kommanditist Gewinnanteile entnimmt, während sein Kapitalanteil durch Verlust unter den Betrag der Haftsumme herabgemindert ist, oder soweit er durch die Entnahme unter die Haftsumme gedrückt wird. $\$ 172$ IV 2 HGB soll sicherstellen, daß der Kommanditist nicht im Wege von Gewinnausschüttungen seine Einlage zurückerhält, obwohl die Einlage zur Verlustabdeckung benötigt wird. $\$ 172$ IV 2 HGB soll mit anderen Worten sicherstellen, daß der Kommanditist, sei es im Rahmen einer unmittelbaren persönlichen Haftung, sei es nach Leistung an die KG, im Rahmen des Gesellschaftsvermögens zumindest einen Einsatz in Höhe der Haftsumme riskiert ${ }^{+2}$; denn die Beteiligung an der KG ist auf längere Zeit angelegt, und erst nach Ablauf dieser Zeit weiß man, ob die Verluste die Gewinne übersteigen werden und ob der Einsatz in Form der Haftsumme zur Abdeckung der überschießenden Verluste benötigt wird. Vertritt man die Ansicht, daß die Haftung nur bis zur Grenze der Haftsumme wiederaufleben kann ${ }^{43}$, so läuft der Sicherungsmechanismus des $\$ 172$ IV 2 HGB zum Teil ins Leere; denn es wird nicht verhindert, daß der Kommanditist durch die Rückgewähr des mehrfachen Betrages seiner Hafteinlage letztlich seinen Einsatz trotz Verluste der KG zurückbekommen kann. Es liegt daher nahe, auf der Grundlage der dem $\$ 172$ iV 2 HGB im manenten Wertung die Rechtsfolge einer Rückgewähr der Einlage dahin abzuändern, daß der Kommanditist immer mit dem Betrag den Gesellschaftsgläubigern erneut zu haften hat, um den sein Kapitalkonto (weiter) unter den Betrag der Haftsumme sinkt.

Diese Lösung gerät jedoch in Konflikt zu der Entscheidung des Gesetzgebers, die KG als Unternehmergesellschaft auszuformen, bei der zumindest eine Unternehmerpersönlichkeit die volle Haftung mit seinem Privatvermögen übernommen hat und bei der in Hinblick auf das

+1 Die Haftung erlischt auch dann wieder, wenn die Einlage in Höhe der Haftsumme erneut erbracht wird (a. A. U.Huber, ZGR 1988, 1, 15; Heymann/Horn, HGB [Fn. 4], \$172 Rdn. 15); denn nur so ist es möglich, die Fälle gleich zu behandeln, in denen der Kommanditist an einen Gesellschaftsgläubiger bzw. an die KG leistet. Die erneute Leistung an die KG hat zur Folge, daß die Haftung erlischt, erneute Auszahlungen, insbesondere des Gewinns, jedoch haftungsschädlich sind, da das Kapitalkonto trotz Leistung negativ geblieben ist.

+2 Vgl. v. Hahn, Commentar zum ADHGB (1863), Bd.1, Art. 165, 7 ; ähnlich Schlegelberger/Geßler (Fn. 4), \$172 Anm. 20.

+3 Siehe oben bei Fn. 7. 
Vermögen des Komplementärs keine Kapitalsicherungsvorkehrungen existieren ${ }^{4+}$.

Auch der Lösungsvorschlag Joosts ${ }^{45}$ ignoriert die Tatsache, daß bei der $\mathrm{KG}$ anders als bei der $\mathrm{GmbH}$ die Gläubiger nicht nur mit Hilfe von Gesellschaftereinlagen, sondern auch mit Hilfe des Privatvermögens des Komplementärs geschützt werden ${ }^{46}$. Je nach dem Umfang des Privatvermögens des Komplementärs treten deshalb die Haftsummen der Kommanditisten mehr oder minder in den Schatten. Man darf daher den typisierenden Gläubigerschutz bei der KG nicht an einem Modell ausrichten, in dem als Komplementär eine Person fungiert, die nichts zu sagen und auch nichts zu verlieren hat ${ }^{47}$. Derart atypische Konstellationen verlangen atypische Lösungen.

Geht man davon aus, daß der typische Komplementär bei der gesetzestypischen KG über Privatvermögen verfügt und etwas zu verlieren hat, so fällt ein weiterer gravierender Unterschied zur $\mathrm{GmbH}$ ins Auge. Bei der GmbH ist das Gläubiger-Gefährdungspotential erheblich höher, weil alle Gesellschafter nur beschränkt haften. Bei der KG wird dagegen der Komplementär, der mit seinem Privatvermögen den Gesellschaftsgläubigern in vollem Umfang haftet, als Bremser fungieren. Er wird, wie der $\mathrm{BGH}^{+8}$ formuliert, den Kapitalwünschen des Kommanditisten im eigenen Interesse verstärkt entgegentreten und auch die Geschäfte der $\mathrm{KG}$ in erhöhtem Maße vorsichtig führen ${ }^{49}$. Dies rechtfertigt es, die Gläubigerschutzvorkehrungen bei der gesetzestypischen KG schwächer als bei der $\mathrm{GmbH}$ auszugestalten. Es ist allerdings zuzugeben, daß diese Bremse aus der Sicht der Gläubiger nicht absolut sicher funktioniert. Der Komplementär kann sich zum Beispiel in Zeiten guter Konjunktur mit Ausschüttungen einverstanden erklärt haben, die der KG in Zeiten einer Rezession zur Bewältigung einer Krise fehlen. Die Bremsfunktion der Komplementärhaftung kann deshalb nicht voll crklären, warum cin Kommanditist aus der KG ein Mehrfaches der Haftsumme abziehen kann, ohne das Risiko eines Wiederauflebens der Haftung in Höhe des empfangenen Betrages zu laufen.

Der entscheidende Grund dafür, daß sich der Gesetzgeber konsequenterweise nur für ein Wiederaufleben der Haftung bis zur Grenze

4t BGHZ 93, 246, 250.

45 Siehe oben bei Fn. 11.

46 Vgl. U.Huber, ZGR 1988, S.1, 14 Fn.37, S. 16, der betont, daß es bei der KG kein Stammkapital gebe. Ebenso Rümker, ZGR 1988, 494, 510; Lutter/Hommelhoff, ZGR $1979,31,45$.

47 So aber Joost, ZGR 1987, 370, 394.

4 BGHZ 60, 324, 327 ff; BGH, ZIP 1990, 578, 583.

49 Wiedemann, FS Bärmann (1975), S. 1037, 1038; U.Huber, ZGR 1988, 1, 16; Lutter/Hommelhoff, ZGR 1979, 31, 45. 
der Haftsumme entschieden hat, liegt darin, daß jede Auszahlung, die nicht durch freie Gewinne oder die eingezahlte, unverbrauchte Einlage gedeckt ist, aus der Sicht der Gläubiger auch eine Auszahlung zu Lasten des Privatvermögens des Komplementärs ist und daß es keinen Kapitalsicherungsmechanismus zu Lasten des Komplementärs gibt.

Das den Gläubigern haftende Vermögen setzt sich bei der KG aus dem Privatvermögen des Komplementärs, dem Gesellschaftsvermögen der KG sowie dem Privatvermögen der Kommanditisten in Höhe der nicht geleisteten bzw. zurückgewährten Haftsumme zusammen. Dieses Vermögen mindert sich, wenn der Komplementär aus seinem Privatvermögen Leistungen an den Kommanditisten erbringt. Obwohl die Gläubigerbefriedigungschancen dadurch sinken, ist diese Transaktion wirksam; sie vergrößert auch nicht die gesellschaftsrechtliche Haftung des Kommanditisten, der die Leistung empfangen hat. Denn, wie der $\mathrm{BGH}^{50}$ formuliert hat: Das Vermögen des voll haftenden Gesellschafters ist dem Zugriff der Gesellschaftsgläubiger nur in seinem jeweiligen Bestand ausgesetzt; es unterliegt keinem gesetzlichen Kapitalerhaltungsgrundsatz. Es kann daher ohne nachteilige Haftungsfolgen zugunsten eines Kommanditisten eingesetzt werden, sofern damit nicht im Einzelfall eine gezielte Gläubigerbeeinträchtigung verbunden ist oder ein Anfechtungstatbestand im Sinn der KO bzw. des AnfG erfüllt ist.

Geht man davon aus, daß der Komplementär i.S.d. $\$ 172$ IV HGB haftungsunschädlich Teile seines Vermögens auf den Kommanditisten verlagern darf, so ist wirtschaftlich gesehen die Situation der Gläubiger sogar besser, wenn der über die Haftsumme hinausgehende Betrag dem Kommanditisten statt aus dem Privatvermögen des Komplementärs aus dem Vermögen der KG zufließt, weil dann den Gläubigern immerhin das Wiederaufleben der Haftung in Höhe der Haftsumme zugute kommt. Es wäre wertungswidersprüchlich, einen Kommanditisten bei der Auszahlung aus dem Privatvermögen des Komplementärs gänzlich ungeschoren zu lassen, bei der Zahlung aus dem Gesellschaftsvermögen dagegen mangels ausreichend aktivem Kapitalkonto über die Haftsumme hinaus in voller Höhe haften zu lassen.

Man wird einwenden, daß Zahlungen aus dem Privatvermögen des Komplementärs die seltene Ausnahme bleiben werden. Bei diesem Einwand wird jedoch verkannt, daß die gesetzestypische KG heute vor allem im Bereich der Familiengesellschaften verbreitet ist. Dort ist es keineswegs ein Einzelfall, daß Vermögenswerte aus dem Privatvermö-

so BGHZ 93, 246, 250; ebenso Baumbach/Duden/Hopt, HGB (28. Aufl.), $\$ 172$ Anm. 2 B; Staub/Schilling, HGB (Fn. 4), $\$ 172$ Rdn. 13; Schlegelberger/K. Schmidt, HGB (Fn. 5), $\$ \$ 171,172$ Rdn. 71 jeweils m. Nachw. zum Streitstand. 
gen oder dem Gesellschaftsvermögen an Kinder des Komplementärs fließen, die in der Rolle als Kommanditisten der KG angehören. Diese stellen sich darauf ein, daß sie die ihnen zugewendeten Beträge behalten dürfen und nicht viele Jahre nach der Zuwendung unter Berufung auf $\$ 172$ IV HGB im Konkurs der KG in Anspruch genommen werden können. In diesem Zusammenhang fällt die $\$ 32 \mathrm{KO}$ zugrundeliegende Wertung ins Gewicht, die zeigt, daß selbst bei unentgeltlichen Zuwendungen die Inanspruchnahme des Empfängers nur in eng limitierten Zeiträumen möglich sein soll.

Auch das Argument, daß im Vergleich zu Zuwendungen aus dem Privatvermögen des Komplementärs ein erhöhter Schutz der Gläubiger vor Zahlungen aus dem Gesellschaftsvermögen deshalb angebracht sei, weil der Komplementär eher geneigt sein werde, das KG-Vermögen als sein Privatvermögen zu plündern, vermag nicht ganz zu überzeugen. Jeder Komplementär, der nicht eine bloße Galionsfigur ${ }^{51}$ darstellt, weiß, daß er für Lücken im Gesellschaftsvermögen mit seinem Privatvermögen aufkommen muß. Er weiß, daß die Gefahr einer Haftung mit seinem Privatvermögen um so geringer ist, je größer das KG-Vermögen ist. Er weiß deshalb auch, daß jede Ausschüttung von Gesellschaftsvermögen sein Risiko, mit seinem Privatvermögen geradestehen zu müssen, verstärkt. Der Komplementär hat daher keinen Anlaß, Ausschüttungen aus dem Gesellschaftsvermögen leichtherziger als Leistungen aus seinem Privatvermögen zuzustimmen. Berücksichtigt man außerdem, daß die Ausschüttung aus dem Gesellschaftsvermögen mit einer Änderung der Beteiligungsverhältnisse verbunden sein kann, so ist aus der Sicht des Komplementärs eine Ausschüttung aus dem Privatvermögen und eine Ausschüttung aus dem Gesellschaftsvermögen häufig gleichwertig. $\mathrm{Ob}$ es zum einen oder zum anderen kommt, ist vielfach deshalb nur eine Frage der Liquidität der jeweiligen Vermögensmassen.

Schließlich ist zu beachten, daß den Gläubigern einer KG nicht garantiert ist, daß nur sie allein in das der Haftung unterworfene Vermögen vollstrecken können. Dies ist zwar in Hinblick auf das Gesellschaftsvermögen der Fall, nicht aber in Hinblick auf das Privatvermögen des Komplementärs und auch nicht in Hinblick auf das Vermögen von Kommanditisten, das den Gläubigern gemäß $\$ 171$ HGB unmittelbar haftet. Es wäre deshalb verfehlt, eine erhöhte Haftung der Kommanditisten mit dem Argument zu rechtfertigen, es müsse der Anreiz vermindert werden, das Vermögen der KG auszuschütten. Das Gesetz statuiert eben im Unterschied zur $\mathrm{GmbH}$ kein Auszahlungsverbot ${ }^{52}$,

\$Vgl. dazu unten bei Fn. 54.

52 Vgl. Schlegelberger/K.Schmidt, HGB (Fn. 5), \$\$171, 172 Rdn. 62. 
sondern motiviert den Kommanditisten nur sanft durch seine unmittelbare Haftung, der KG seine Einlage zu erbringen ${ }^{53}$. Erst recht statuiert das Gesetz kein Auszahlungsverbot in Richtung auf den Komplementär. Von einem den Gläubigern gesichert zugewiesenen Gesellschaftsvermögen kann daher nicht die Rede sein. Auch dies hat zur Konsequenz, daß sich die Gläubiger bei Zuwendungen über die Haftsumme hinaus letztlich in Hinblick auf die spezifische Kommanditistenhaftung so behandeln lassen müssen, als ob der Komplementär aus seinem Privatvermögen geleistet hätte. Eine Parallele zu den $\$ \mathbb{S} 30 \mathrm{ff} \mathrm{GmbHG}$ scheidet aus.

Insgesamt zeigt sich mithin, daß der Kommanditist bei einer gesetzestypischen KG grundsätzlich nicht in Parallele zu den $\$ \$ 30 \mathrm{ff}$ GmbHG über die Haftsumme hinaus für empfangene Leistungen einzustehen hat. Zugleich ergeben sich aber auch aus den Gründen, die dazu führen, daß der Kommanditist maximal bis zur Grenze der Haftsumme haftet, die Kriterien, anhand derer dieser Grundsatz einzuschränken ist. Danach haftet der Kommanditist unabhängig davon, ob eine Gläubigergefährdung i.S.d. $\ 826$ BGB nachgewiesen werden kann oder ob die Voraussetzungen einer Konkursanfechtung i. S.d. $\mathbb{S} 30 \mathrm{ff} \mathrm{KO}$ gegeben sind, wenn der Komplementär nach der Gesellschaftsverfassung nichts zu sagen hat und aus der Sicht des Kommanditisten erkennbar nichts mehr - zu verlieren hat ${ }^{54}$. Dann wirkt nämlich weder die Bremsfunktion der Komplementär-Rolle, noch läßt sich sagen, daß die die Haftsumme übersteigenden Ausschüttungen wirtschaftlich betrachtet letztlich aus dem ungebundenen Privatvermögen des Komplementärs aufgebracht werden. Gleiches muß gelten, falls der Komplementär aus irgendwelchen Gründen, z. B. aus Gründen verwandtschaftlicher Nähe, ersichtlich seine Bremsfunktion nicht erfüllen kann und aus der Sicht des Kommanditisten erkennbar ist, daß die Lücke im Gesellschaftsvermögen durch die Ausschüttung zugunsten des Kommanditisten in absehbarer Zeit nicht durch das Privatvermögen des Komplementärs gedeckt ist. In diesen Fällen lebt die Haftung nicht nur bis zur Grenze der Haftsumme, sondern in voller Höhe des Betrages wieder auf, um den das Kapitalkonto (weiter) unter die Haftsumme ${ }^{55}$ gedrückt wird. Ist die Situation so, daß das Privatvermögen des Komplementärs einen Teil der Ausschüttung deckt, so ist der Betrag, um den die Haftung über die Haftsumme hinaus wieder auflebt, um diesen Teil zu kürzen.

53 Wiedemann, FS Bärmann (Fn. 49), S. 1038.

5t Vgl. Wiedemann, FS Bärmann (1975), S. 1037, 1049f; Blaurock, FS Stimpel (1985), 553, 569; U. Huber, ZGR 1988, 1, 17.

5 Haftsumme des Kommanditisten, der die Leistung empfangen hat. U. Huber, ZGR 1988, 1, 14 Fn. 37 hat überzeugend dargetan, daß nicht auf die Gesamtheit der Haftsummen abgestellt werden kann. 
Auf diese Weise läßt sich auch die Brücke zur Rechtsprechung zu der GmbH \& Co KG schlagen. Der BGH ${ }^{56}$ hat vor kurzem entschieden, daß auch der Nur-Kommanditist für Ausschüttungen in einer $\mathrm{GmbH} \& \mathrm{Co}$ $\mathrm{KG}$ analog den $\mathbb{\$} 30,31 \mathrm{GmbHG}$ einzustehen hat, wenn die Ausschüttung zur Folge hat, daß dadurch mittelbar das Vermögen der Komplementär-GmbH unter den Nennwert des Stammkapitals herabsinkt. Der BGH begründet die Ausdehnung der Haftung des Kommanditisten über die Haftsumme hinaus damit, bei einer $\mathrm{GmbH}$ als Komplementär sorge die unmittelbare Haftung nicht in gleichem Maß wie bei der unmittelbaren Haftung natürlicher Personen dafür, daß das Unternehmen wirtschaftlich geführt wird und untragbare Vermögensaushöhlungen unterbleiben. Diese Argumente stellen nur die eine Seite der Medaille dar. Das Bild wird vollständig, wenn man bedenkt, daß man bei der $\mathrm{GmbH}$ eben nur über das Vermögen, das nicht zur Deckung der echten Passiva und des Stammkapitals benötigt wird, so frei verfügen kann wie über das Privatvermögen einer natürlichen Person. Soweit kein freies Vermögen vorhanden ist, ist die Situation dieselbe wie in Fälllen, in denen eine Privatperson auf absehbare Zeit faktisch vermögenslos ist.

\section{Eigenkapitalersetzende Darlehen}

Definiert man als faktisch eigenkapitalersetzende Darlehen all diejenigen Darlehen, die von Gesellschaftern zu einem Zeitpunkt vergeben werden, in dem kein außenstehender Kreditgeber bei Kenntnis aller Umstände mehr ein Darlehen zu marktüblichen Bedingungen gewährt hätte und die Gesellschaft deshalb hätte liquidiert werden müssen ${ }^{57}$, so stellen eigenkapitalersetzende Darlehen sicherlich keine Besonderheit der GmbH sowie der GmbH \& Co KG dar, sondern sind Erscheinungen, die bei allen Gesellschaften, ja Unternchmen, anzutreffen sind ${ }^{58}$. Es erhebt sich deshalb die Frage, was es rechtfertigt, die Sonderregeln, die im Rahmen der $\mathrm{GmbH}$ und $\mathrm{GmbH} \& \mathrm{CoKG}$ entstanden sind und vom Gesetzgeber zum Teil festgeschrieben worden sind (\$\$32a GmbHG, 172 a HGB), nicht auf Kommanditgesellschaften mit einer voll haftenden natürlichen Person als Gesellschafter unmittelbar oder modifiziert zu übertragen.

Der angebliche Selbstwiderspruch ${ }^{59}$, der in der Vergabe eigenkapitalersetzender Darlehen liegt, genügt sicherlich nicht, denn niemand kann darauf vertrauen, daß ein Kommanditist in einer Krise statt Darlehen Eigenkapital nachschieß̊t oder Darlehen, die er nicht abzieht, mit vollem

\footnotetext{
56 BGH, ZIP 1990, 578, 583.

5) K.Schmidt, Gesellschaftsrecht (1986), S. 395.

5s $\mathrm{Vgl}$. oben bei Fn. 13.

59 Vgl. Fn. 20.
} 
Verlustrisiko stehenläßt ${ }^{60}$. Für eine Gleichbehandlung der eigenkapitalersetzenden Darlehen bei allen Gesellschaftsformen spricht vielmehr die Finanzierungsverantwortung der Gesellschafter ${ }^{61}$, die grundsätzlich auch einen gesetzestypisch beteiligten Kommanditisten trifft. Das Schlagwort „Finanzierungsverantwortung" ist im Licht der Funktionen zu konkretisieren, die die Figur der eigenkapitalersetzenden Darlehen zu erfüllen hat: Ziel der weitgehenden Gleichstellung von eigenkapitalersetzenden Darlehen und echtem Eigenkapital ist es, die Gesellschafter zu veranlassen, in Zeiten, in denen Dritte bei Kenntnis aller Umstände keinen Kredit mehr zu marktüblichen Bedingungen gewähren würden, und die Gesellschaft deshalb liquidiert werden müßte, nur dann Kapital nachzuschießen oder es stehenzulassen, wenn sie bereit sind, das Kapital wie Eigenkapital voll zu riskieren. Die Bereitschaft zum vollen Einsatz fungiert demnach als Prüfstein, an dem sich zeigt, ob wenigstens die Gesellschafter an einen hinreichend erfolgversprechenden Weg aus der Krise glauben. Tun sie dies und bringen sie weiter Eigenkapital ein, so kann man annehmen, daß Alt- und Neugläubiger, die den wahren Stand des Unternehmens nicht so gut kennen, nicht derart gefährdet sind, daß sie durch eine alsbaldige Liquidation des Unternehmens vor einem weiteren Schwund des Gesellschaftsvermögens geschützt werden müßten. Die Figur der Finanzierungsverantwortung knüpft mithin an typische Informationsvorsprünge der Gessellschafter als Insider $\mathrm{an}^{62}$ und an die Möglichkeit der Gesellschafter, das Fortleben der Gesellschaft, den Geldfluß und Unternehmenserfolg zu beeinflussen ${ }^{63}$. Die Vorstellung von einer Finanzierungsverantwortung orientiert sich mithin an der Korrelation von Herrschaft und Haftung. Außerdem spielt das besondere Vertrauensverhältnis eine Rolle, das Gesellschafter typischerweise miteinander verbindet, weil es dazu beiträgt, daß bei einem sich abzeichnenden Zusammenbruch die Ansprüche der Gesellschafter gegen die Gesellschaft mit Vorrang vor sonstigen Gläubigern befriedigt werden.

Aus dieser Perspektive läßt sich nicht abstreiten, daß auch der gesetzestypisch beteiligte Kommanditist in der Lage ist, den Unternehmenserfolg zu beeinflussen $(\$ 164 \mathrm{HGB})^{64}$. Ein gesetzestypischer Kommanditist steht außerdem üblicherweise den anderen Gesellschaftern so

${ }_{60}$ Zutreffend K. Schmidt, Gesellschaftsrecht (1986), S. 394.

61 BGHZ 90, 381, 388; BGH, ZIP 1990, 578, 584; Rümker, ZGR 1988, 494; K. Schmidt Gesellschaftsrecht (1986), S. 395.

62 Die Informationsvorsprünge sind nicht allein ausschlaggebend; BGHZ 76, 326, 330.

6. Es bleibt offen, ob die Finanzierungsverantwortung erst ab $10 \%$ Gesellschaftsanteil zu entstehen beginnt. Vgl. Rümker, ZGR 1988, 494, 504.

6+ Vgl. Rümker, ZGR 1988, 494, 513 mit anderem dogmatischen Ansatz, der die Schwäche aufweist, daß er dem Geschick der Kautelarjuristen ausgeliefert ist. 
nahe, daß er damit rechnen kann, daß seine Forderungen vorrangig aus dem Gesellschaftsvermögen bedient werden. Problematisch ist nur die Antwort auf die Frage, ob ein gesetzestypisch beteiligter Kommanditist auch als typischer Insider qualifiziert werden $\mathrm{kann}^{65}$. Stünden einem Kommanditisten nur Informationsmöglichkeiten wie jedem außenstehenden Gläubiger zur Verfügung, so wäre es wertungswidersprüchlich, den Kommanditisten schärfer als etwa ein Kreditinstitut haften zu lassen $^{66}$. Die Kontrollrechte, die dem Kommanditisten gemäß $\$ 166$ HGB zugewiesen sind, sprechen zwar auf den ersten Blick dafür, ihn als Insider einzuordnen. Es muß jedoch auch berücksichtigt werden, daß die Kreditwürdigkeit der KG nicht nur von den Vermögensverhältnissen der KG selbst, sondern auch von den Vermögensverhältnissen des Komplementärs abhängt. Der Kommanditist hat indessen nur in die Vermögensverhältnisse der KG Einblick, nicht aber auch in die des Komplementärs. In Hinblick auf das Privatvermögen des Komplementärs steht der Kommanditist grundsätzlich einem normalen Gläubiger gleich $^{67}$. Fungiert eine juristische Person als Komplementär, so ist die Situation dort anders, wo die Anteile der juristischen Person bei der KG liegen; denn dann kann der Kommanditist in die Bilanzen der juristischen Person Einblick nehmen. Aber auch in anderen Fällen der Beteiligung einer juristischen Person an der KG vermag der Kommanditist die Entwicklung des Vermögens des Komplementärs aus einer etwaigen gleichzeitigen Beteiligung beim Komplementär oder in gewissem Umfang aus publizierten Bilanzen ${ }^{68}$ zu beobachten. Die generalisierende Unterwerfung der Kommanditisten einer KG, die keine natürliche Person als Komplementär aufweist, unter die Regeln des eigenkapitalersetzenden $\mathrm{GmbH}-G e s e l l s c h a f t e r d a r l e h e n s^{69}$ steht daher im allgemeinen mit dem Grundsatz im Einklang, daß eine Behandlung der eigenkapitalersetzenden Darlehen als nachrangiges Eigenkapital die ausreichende Information des darlehensgewährenden Gesellschafters über die mangelnde Kreditwürdigkeit der KG voraussetzt ${ }^{70}$. Bei KGs, bei denen eine natürliche Person den Komplementär stellt, läßt sich dagegen nicht typisierend sagen, daß Kommanditisten über den Stand des Komplementärvermögens ausreichend informiert sind. Eine Anwendung der Sonderregeln

65 Zweifelnd-H.P. Westermann, FS Fleck (Fn. 1), S. 436.

66 Nach BGHZ 76, 326, 330 stellt die Insiderposition freilich keinen ausreichenden Grund für die Sonderbehandlung von Gesellschafterdarlehen dar.

67 Zum Beispiel in Hinblick auf Ausgleichsansprüche bei Liquidation.

$6 s \int S 325 \mathrm{ff} \mathrm{HGB}$.

69 Über den $\$ 172$ a HGB hinaus; vgl. BGH, ZIP 1990, 578, 583.

70 Der BGH, ZIP 1990, 578, 583 geht allerdings auf das Informationsproblem nicht ein und postuliert pauschal eine Finanzierungsverantwortung des Kommanditisten. 
über eigenkapitalersetzende Darlehen ist deshalb nur gerechtfertigt, wenn im Einzelfall nachgewiesen werden kann, daß der Kommanditist wußte oder wissen mußte, daß der Komplementär nicht - mehr - über ausreichendes Aktivreinvermögen verfügt, um die Kreditwürdigkeit der KG zu sichern.

Kann dieser Beweis geführt werden, so müssen die vom Kommanditisten in der Krise gegebenen oder stehengelassenen Darlehen wie Eigenkapital behandelt werden. $₫ 32$ a GmbHG enthält insoweit einen auch für die gesetzestypische $\mathrm{KG}$ maßgeblichen Grundsatz.

Ist das Darlehen schon vor Konkurseröffnung an den Kommanditisten zurückgezahlt worden, so stellt sich die Frage, ob auch $\$ 32$ a KO oder ob, wie Karsten Schmidt $t^{71}$ vorschlägt, $\$ 237$ HGB analog anzuwenden ist. Die Analogie zu $\$ 237$ HGB weist die Schwäche auf, daß der Rückgewähranspruch von einer besonderen Vereinbarung ${ }^{72}$ der Gesellschafter über die vorzeitige Rückgewähr des eigenkapitalersetzenden Darlehens abhängen würde. Diese Voraussetzung ist bei all den Darlehen nicht erfüllt, die gekündigt werden konnten und fristgerecht gekündigt worden sind.

Es ist daher primär eine Analogie zu $₫ 32 \mathrm{a} \mathrm{KO}$ zu prüfen. Dieser Analogie läßt sich nicht das Argument entgegenhalten, der Kommanditist müsse ja auch bei der Rückgewähr von Vermögenswerten, auf die er keinen Anspruch besitzt oder die aus seiner Pflichteinlage stammen, maximal nur mit einem Wiederaufleben der persönlichen $\mathrm{Haftung}$ bis zur Grenze der Haftsumme rechnen ${ }^{73}$. Dieses Argument ist nämlich nur tragfähig, wenn diejenigen Gründe, die für ein beschränktes Wiederaufleben der Haftung bei der Rückgewähr der Einlage sprechen, auch im Fall der Rückzahlung eigenkapitalersetzender Darlehen eine Rolle spielen.

Derartige Gründe lassen sich bei näherer Untersuchung nicht immer feststellen: Soll zu einem Zeitpunkt, in dem die Gesellschafter zu dem Ergebnis gelangt sind, daß sich ein Niedergang der Gesellschaft aus eigenen Kräften der KG und des Komplementärs nicht mehr aufhalten läßt, ein eigenkapitalersetzendes Darlehen zurückgezahlt werden, so wird der Komplementär trotz seiner unbeschränkt persönlichen Haftung kaum mehr zugunsten des Gesellschaftsvermögens als Bremser fungieren; denn er muß sich sagen, daß er nichts mehr zu verlieren hat. Aus seiner Sicht ist es nämlich gleichgültig, ob er sich den Ansprüchen des Kommanditisten als Fremdkapitalgeber oder bei einer Umqualifikation des Darlehens in nachrangiges Eigenkapital Ansprüchen im Rahmen

7 K. Schmidt, GmbHRdSch. 1986, 337, 342.

72 Schlegelberger/K.Schmidt, HGB (Fn. 5), $\$ 342$ (\$237 n. F.) Rdn. 6.

${ }^{73}$ Siehe oben bei Fn. 41. 
der Liquidation bzw. des Konkurses ( $\$ \$ 161$ II, 105 II HGB, 735 BGB) ausgesetzt sieht. Der Kommanditist, an den das, wie er wissen muß, eigenkapitalersetzende Darlehen in der Krise zurückgewährt wird, wird auch nicht immer darauf vertrauen dürfen, daß ihm wirtschaftlich gesehen letztlich ungebundenes Privatvermögen des Komplementärs zufließt. An einer derartigen Vertrauensgrundlage fehlt es, wenn der Kommanditist wissen muß, daß Privatvermögen des Komplementärs, aus dem das Darlehen zurückgezahlt werden könnte, nicht zur Verfügung steht. Unter dieser Voraussetzung steht einer Analogie zu $\ 32$ a $\mathrm{KO}$ nichts im Wege.

Anders ist die Situation, wenn der Kommanditist annehmen konnte, daß der ihm zurückgezahlte Betrag aus dem Privatvermögen des Komplementärs hätte geleistet werden können. Dann müssen sich die Gläubiger mit der Tatsache abfinden, daß bei der KG kein gesichertes Kapital, vor allem kein gesichertes Privatvermögen des Komplementärs existiert und daß der Komplementär nach dem Recht der KG haftungsunschädlich die Einlage des Kommanditisten aus seinem Privatvermögen zurückzahlen kann $^{74}$. Dieser Umstand steht, wie Karsten Schmidt ${ }^{75}$ zutreffend herausgearbeitet hat, auch einer Analogie zu den $\mathbb{\$} 30,31$ GmbHG entgegen. Der Schutz der Gläubiger gegen den Abfluß des ungebundenen Komplementär-Privatvermögens kann dann ausschließlich auf die $\$ \$ 826 \mathrm{BGB}, 30-32 \mathrm{KO}$, auf das AnfG und eventuell auf eine c. i. c. ${ }^{76}$ gestützt werden.

\section{Stille Beteiligung}

Joost ${ }^{77}$ und Schön ${ }^{78}$ haben zu Recht betont, daß die Qualifikation von stillen Einlagen als normales Eigenkapital, wie sie der $\mathrm{BGH}^{79}$ bei gesplitteten Einlagen vornimmt, einen untragbaren Verstoß gegen die Privatautonomie darstellt. Damit soll nicht abgestritten werden, daß unter den Voraussetzungen, unter denen Darlehen als eigenkapitalersetzende Darlehen zu betrachten sind ${ }^{80}$, stille Einlagen als nachrangiges, aber eben nur als nachrangiges Eigenkapital anzusehen sind. Sie sind nach den hier für die eigenkapitalersetzenden Darlehen entwickelten Regeln zu behandeln ${ }^{81}$.

\footnotetext{
${ }^{74}$ Siehe oben bei Fn. 50.

75 K.Schmidt, AG 1984, 12, 14; GmbHRdSch. 1986, 337, 340.

76 Vgl. Canaris in: Freiheit und Zwang, FS Giger (1989), S. 91, $119 \mathrm{ff}$.

77 Siehe oben Fn.1.

78 Siehe oben bei Fn. 26.

79 Vgl. Fn. 1.

so Siehe oben bei Fn. 70.

si Vgl. Joost, ZGR 1987, 370, 399.
} 
Die von $S c h o ̈ n^{\$ 2}$ propagierten, darüber hinausgehenden Vorschläge sind dagegen unhaltbar. Schön ${ }^{83}$ arbeitet mit einer empirisch in keiner Weise abgesicherten Verkehrsauffassung, derzufolge die bekannt gewordene stille Einlage selbst dann als Haftungszusage anzusehen ist, wenn der stille Gesellschafter zugleich offenlegt, daß er am Verlust nicht beteiligt sein will. Auch unter normativen Aspekten ist die These von einer Haftungszusage nur haltbar, wenn man ausschließlich vom gewünschten Ergebnis her denkt. Das Gesetz jedenfalls sieht nicht in jeder offenbarten Beteiligung an einem Unternehmen eine Haftungszusage. Dies gilt nicht nur für die stille Beteiligung am Unternehmen des Einzelkaufmanns $s^{84}$, sondern auch für die stille Beteiligung an einer Gesellschaft; denn \172 I HGB stellt aus Gründen der Rechtssicherheit ${ }^{85} \mathrm{klar}$, daß grundsätzlich nur die im Handelsregister eingetragene Beteiligung eine Art Haftungszusage darstellt. Auf die höhere Pflichteinlage dürfen sich die Gesellschaftsgläubiger nämlich selbst dann nicht berufen, wenn sie ihnen bekannt geworden ist. Dies ergibt sich im Umkehrschluß aus $₫ 172$ II HGB, demzufolge den Gläubigern sogar eine im Handelsregister nicht eingetragene Erhöhung der Haftsumme nur dann zugute kommt, wenn sie ihnen gezielt ${ }^{86}$ mitgeteilt oder in handelsüblicher Weise bekanntgemacht worden ist. Diese im Interesse der Rechtssicherheit liegende Typisierung würde man aufgeben, wenn man von Fall zu Fall prüfen müßte, ob ein verständiger Gläubiger die Information über eine stille Beteiligung an einer KG als Haftungszusage verstehen darf. Erst recht kann die Offenlegung der Beteiligung nicht zugunsten aller Gläubiger wirken, wenn man gleichzeitig im Rahmen des $\$ 172$ II HGB die Ansicht vertritt, daß eine Mitteilung der nicht eingetragenen Erhöhung der Haftsumme nur denjenigen Gläubigern Ansprüche eröffnet, denen die Mitteilung gezielt zugegangen ist ${ }^{87}$. Auch die Parallele zur Zahlung von Einlagen bei negativem Kapitalkonto ${ }^{\text {ss }}$ vermag nicht zu überzeugen. Wenn der Kommanditist Leistungen, die er zusätzlich ohne Erhöhung der Haftsumme als Einlage erbracht hat, nicht haftungsunschädlich zurückholen darf, nachdem sie mit Verlusten oder Entnahmen auf seinem negativen Kapitalkonto verrechnet worden sind,

\$2 Siehe oben bei Fn. 26.

83 Schön, ZGR 1990, 220, 239.

st So auch Schön, ZGR 1990, 220, 237.

5 Denkschrift (Fn. 2), S. 285: Es bedarf, weil die Einlage im Innen- anders als im Außenverhältnis festgesetzt werden könne, einer „für Jedermann kenntlichen Festsetzung, aus der die Höhe der Einlage..." zu entnehmen sei.

86 Staub/Schilling, HGB (Fn. 4), $\$ 172$ Rdn. 5 m. Nachw.

s7 Allgem. Meinung; Schlegelberger/K.Schmidt, HGB, $\$ \$ 171,172$ Rdn.37 m. Nachw.

ss Siehe oben bei Fn. 29. 
so beruht dies zum einen auf seiner Entscheidung, sie als Kommanditeinlage zur Verrechnung zu stellen. Zum anderen kann der Rechtssicherheit wegen ${ }^{89}$ die Verrechnung nicht mehr rückgängig gemacht werden, weil sonst rückwirkend festgestellt werden müßte, ob die Auszahlung von Gewinnen haftungsschädlich war und ob Gläubiger einen unmittelbaren Anspruch gegen den Kommanditisten ( $\$ \mathbb{S} 172$ IV 2, 171 I HGB) erworben hatten. Ein rückwirkendes Wiederaufleben der unmittelbaren Haftung ist aber wenig sinnvoll. Deshalb ist eine Kommanditeinlage, die mit Verlusten oder Entnahmen verrechnet worden ist, endgültig verbraucht. Von einem derartigen Verbrauch läßt sich bei einer stillen Einlage indessen nur insoweit sprechen, als der Stille eine Verlustbeteiligung zugesagt hatte. Wurde eine vollkommen stille Beteiligung ohne Verlustbeteiligung vereinbart und praktiziert, so besteht kein Anlaß, die Gläubiger nur deshalb besser zu stellen, weil die Einlage von einem Kommanditisten stammt. Es steht dem Kommanditisten grundsätzlich frei zu entscheiden, welches Risiko er übernimmt. Für den nötigen Schutz der Gläubiger sorgen die analog anwendbaren Regeln über die Behandlung eigenkapitalersetzender Darlehen und $\$ 237$ HGB.

\section{Ergebnisse}

1. Eine die Haftungssumme übersteigende Einlage kann, soweit sie nicht durch Verluste verbraucht ist, haftungsunschädlich zurückgezahlt werden.

2. Die Haftung aus $\mathbb{S} 172 \mathrm{IV}, 171 \mathrm{I}$ HGB lebt auch bei Leistungen, die die Haftsumme übersteigen, nur bis zum Betrag der Haftsumme wieder auf. Das gilt nicht, wenn der Kommanditist erkennen konnte, daß das Privatvermögen des Komplementärs nicht den Abfluß des Gesellschaftsvermögens ausgleicht und der Komplementär dem Abfluß keinen hinreichend starken Widerstand entgegensetzten kann.

3. Eigenkapitalersetzende Darlehen sind im Konkurs der KG als nachrangiges Eigenkapital zu behandeln, wenn der Kommanditist zumindest wissen mußte, daß der Komplementär nicht mehr über ausreichendes Privatvermögen verfügte. $\$ 32 \mathrm{a} \mathrm{KO}$ ist analog anzuwenden.

4. Stille Einlagen begründen keine Haftungszusage gegenüber den Gläubigern. Sie sind unter den für eigenkapitalersetzende Darlehen geltenden Voraussetzungen wie derartige Darlehen zu behandeln. 